\title{
The floating point: tales of the unexpected
}

\author{
David A. Faux* and Janet Godolphin \\ Faculty of Engineering and Physical Sciences, \\ University of Surrey, Guildford, GU2 7XH, UK
}

(Dated: March 8, 2021)

\begin{abstract}
Digital computation is central to almost all scientific endeavor and has become integral to university physics education. Students collect experimental data using digital devices, process data using spreadsheets and graphical software, and develop scientific programming skills for modeling, simulation and computational work. Issues associated with the floating-point representation of numbers are rarely explored. In this article, problems of floating point are divided into three categories: significant-figure limits, propagation of floating-point representation error, and rounding. For each category, examples are presented of unexpected ways in which the digital representation of floating-point numbers can impact the veracity of scientific results. These examples cover aspects of classical dynamics, numerical integration, cellular automata, statistical analysis, and digital timing. Suggestions are made for curriculum enhancement and project-style investigations that reinforce the issues covered at a level suitable for physics undergraduate students.
\end{abstract}




\section{INTRODUCTION}

Digital processing is ubiquitous in scientific investigation. All numerical computation, computer simulation, processing of experimental data, and even gathering of the experimental data itself, normally utilise digital processing. Almost all digital computers use a sequence of binary digits (bits) to represent decimal (base 10) floating-point numbers. Typically 32 bits are used to represent a number to single precision and 64 bits to doubleprecision. For example, an IEEE floating-point number reserves one bit for the sign of the number, 8 bits for the exponent and 23 bits for the mantissa. ${ }^{1}$ An IEEE double-precision floating-point number is constructed in a similar fashion but with 11 and 52 bits for the exponent and mantissa respectively. Issues of the limitation of significant figures and of rounding have been discussed from the late 1950's when numerical computation started to become widespread (see $\mathrm{Grau}^{2}$, Wilkinson ${ }^{3}$ and sources therein) and a clear and detailed description of the IEEE single- and double-precision representation of numbers and precision limitations are presented by Landau et al. ${ }^{4}$.

Basic concepts of computer programming are taught in high school and programming forms a key part of the physics degree curriculum. ${ }^{5}$ Physics undergraduate curricula typically include a staged development of programming skills, and students have the opportunity to undertake extensive project work in later years. Diffusion, magnetism, thermodynamics, classical dynamics and financial modeling are amongst many subject areas that lend themselves to numerical computational investigation. Such work provides the dual benefits of reinforcing understanding of the subject area and developing skills in data analysis. ${ }^{6-9}$ Popular programming languages are Python and Matlab with many excellent student guides available. ${ }^{10,11}$

The development of numerical simulation courses increased significantly from about 2008 as the popularity of Python, in particular, took hold. ${ }^{12-16}$ Classical dynamics provides a suitable topic for initial computational investigation. Indeed, in the first semester of year 1, physics undergraduates at the University of Surrey engage in elementary dynamical modeling of the trajectory of a fictional asteroid using a spreadsheet. ${ }^{17}$ In subsequent semesters, students tackle numerical integration, thermodynamic modeling and a simulation project, all using Python. The inclusion of computational physics develops programming skills and reinforces the core curriculum. 
Undergraduate work in numerical simulation should address the issue of floating-point error because it is important to determine which errors are due to algorithmic and computational methods as opposed to limitations in the model itself. Issues of floating-point representation are normally raised in the context of logical comparison. Two floating-point numbers a and $\mathrm{b}$ should not be tested for equality using the logic operator $==$, since the result of if $\mathrm{a}==\mathrm{b}$ may or may not yield TRUE if $\mathrm{a}$ and $\mathrm{b}$ are "equal". The problem is overcome by taking the absolute value of the difference and comparing with a suitable small number as in the expression if $\operatorname{abs}(a-b)<0.000001$. Beyond this example, issues with floating-point representation rarely appear and so may be overlooked when they do.

This article is concerned with educating undergraduate physics students in floatingpoint error and highlighting opportunities for curriculum enhancement. Potential issues of floating-point error are well known but nonetheless we show that problems may manifest in interesting and unexpected forms. The first category of problem arises due to the limited accuracy of floating-point numbers due to their representation as a finite bit string. The second arises due to the propagation of floating-point representation error. Rounding errors provide the third class of problem. Each is illustrated by novel examples relevant to the physics undergraduate curriculum.

\section{SIGNIFICANT FIGURE LIMITATIONS}

Computers store floating-point numbers in the form of a sequence of binary digits. The number of binary digits used depends on the desired precision. Software will operate a default precision for floating-point numbers which is typically about 15 significant figures. For example, the scientific programming language FORTRAN allows the coder to specify "single precision" or "double precision" allowing 7 or 15 significant digits of accuracy respectively, whereas Matlab and Python default to 15 significant figures of accuracy but allow higher precision alternatives. ${ }^{18}$

A Matlab code printed the decimal numbers 0.0 to 0.9 , in increments of 0.1 , to 30 significant figures and the ouput is contained in Table I. ${ }^{19}$ The values are accurate to 15 digits (the default for Matlab) except for the numbers 0.0 and 0.5 which are exactly represented to the full 30 significant figures on display. Table II explains how a decimal is converted to binary. At each step, the decimal number is multiplied by two and the integer digit is taken 
TABLE I. The table illustrates the accuracy to which decimals can be represented using Matlab. ${ }^{19}$ The second column contains the decimal representation of the values in column 1 after conversion to binary for storage and then back to base 10 for display, stored to 30 significant figures. For the examples listed, only 0.0 and 0.5 are exactly represented.

\begin{tabular}{cc}
\hline \hline decimal & floating-point value to 30 significant figures \\
\hline 0.0 & 0.000000000000000000000000000000 \\
0.1 & 0.100000000000000005551115123126 \\
0.2 & 0.200000000000000011102230246252 \\
0.3 & 0.299999999999999988897769753748 \\
0.4 & 0.400000000000000022204460492503 \\
0.5 & 0.500000000000000000000000000000 \\
0.6 & 0.599999999999999977795539507497 \\
0.7 & 0.699999999999999955591079014994 \\
0.8 & 0.800000000000000044408920985006 \\
0.9 & 0.900000000000000022204460492503 \\
\hline \hline
\end{tabular}

out. The base 10 number $0.375_{10}$ is represented as $0.011_{2}$. The decimals $0.0_{10}$ and $0.5_{10}$ are represented as $0_{2}$ and $0.1_{2}$ respectively and therefore both can be represented exactly in binary as shown in Table II. By contrast, $0.3_{10}$ is represented by the repeating infinite sequence of binary digits $0.1001100110011 \ldots$ and cannot therefore be represented exactly in the computer. The closest binary representation is used. Fifteen significant figures of accuracy is normally sufficient for numerical computation but there are occasions when limited floating-point precision leads to problems. Two examples are presented, one from a dynamical simulation and one from numerical integration.

\section{A. Computer simulation of planetary orbit}

Much of computational astrophysics research involves the computer simulation of the dynamics of celestial objects (planets, stars, galaxies, black holes, dark matter, etc.). These simulations employ Newton's equations of motion and Newton's law of gravitation using approximate computational techniques iterated through a sequence of time steps. A simulation starts with the calculation of the net gravitational force on each mass. Assuming constant acceleration during time period $\Delta t$, the change in velocity and the displacement of each mass may be computed. The net gravitational force is then recalculated for each mass and the computational procedure is repeated.

We present an illustration of the impact of floating-point error using a model designed to 
TABLE II. The table illustrates how some selected decimal numbers are converted to base 2 . The decimal is multiplied by 2 whereupon the whole number is held and the remainder goes through the repeat process.

\begin{tabular}{|c|c|c|c|c|}
\hline number & calculation & integer & remainder & binary \\
\hline \multirow[t]{3}{*}{0.375} & $0.375 \times 2=$ & 0 & 0.75 & \\
\hline & $0.75 \times 2=$ & 1 & 0.5 & \\
\hline & $0.5 \times 2=$ & 1 & 0 & 0.011 \\
\hline 0.5 & $0.5 \times 2=$ & 1 & 0 & 0.1 \\
\hline \multirow[t]{10}{*}{0.3} & $0.3 \times 2=$ & 0 & 0.6 & \\
\hline & $0.6 \times 2=$ & 1 & 0.2 & \\
\hline & $0.2 \times 2=$ & 0 & 0.4 & \\
\hline & $0.4 \times 2=$ & 0 & 0.8 & \\
\hline & $0.8 \times 2=$ & 1 & 0.6 & \\
\hline & $0.6 \times 2=$ & 1 & 0.2 & \\
\hline & $0.2 \times 2=$ & 0 & 0.4 & \\
\hline & $0.4 \times 2=$ & 0 & 0.8 & \\
\hline & $0.8 \times 2=$ & 1 & 0.6 & \\
\hline & $\ldots$ & $\ldots$ & $\ldots$ & $0.010011001100110011 \ldots$ \\
\hline
\end{tabular}

simulate the trajectory of a fictional asteroid as it moves around the Sun. This example is based on an exercise used in the first year of our undergraduate physics programme. ${ }^{17}$ First, the model is tested on a simulation of the Earth moving in the $x-y$ plane to confirm that the computational procedure is functioning correctly and to appraise its accuracy. Given the Earth's position $(x, y)$ and velocity $\left(v_{x}, v_{y}\right)$ at a specified point in time, the $x$ and $y$ components of the acceleration $\left(a_{x}, a_{y}\right)$ allow the velocity components to be calculated after a time interval $\Delta t$ using Newton's equations. For the $x$ components:

$$
a_{x, i}=\frac{-G M x_{i}}{r_{i}^{3}}
$$

at time step $i$. Similar expressions are obtained for the $y$ components, thus providing the position and velocity of the asteroid at time step $i+1$. The gravitational constant is $G=6.67408 \times 10^{11} \mathrm{~m}^{3} \mathrm{~kg}^{-1} \mathrm{~s}^{-2}$, the Sun mass $M=1.989 \times 10^{30} \mathrm{~kg}$ and $r^{2}=x^{2}+y^{2}$. The new 
forces acting on each mass are calculated and the cycle is repeated. However, the algorithm provided by Eq. (1) to Eq. (3) is poor. This is demonstrated, for example, by the choice of the time step $\Delta t=1$ day: the Earth gradually spirals away from the sun and total energy is not conserved.

Implementation of a velocity Verlet algorithm, similar to that used in dynamical simulations from atomistic to astrophysical scales, significantly improves the overall accuracy of the algorithm. ${ }^{20}$ In the velocity Verlet algorithm, Eq. (3) is replaced by

$$
v_{x, i+1}=v_{x, i}+\frac{1}{2}\left(a_{x, i}+a_{x, i+1}\right) \Delta t .
$$

A FORTRAN program was written that demonstrates: the role of the choice of $\Delta t$ in the accuracy of the simulation; the importance of significant figures of accuracy in dynamical simulation, and also the impressive performance of the velocity Verlet algorithm. ${ }^{19}$ The Earth was assumed to move in a perfect circular orbit of radius $R_{E}=1.5 \times 10^{11} \mathrm{~m}$ around the Sun located at the origin. It is this idealised orbit that the numerical simulation sought to emulate. The Earth was placed at starting position $\left(R_{E}, 0\right)$ with initial velocity components $v_{x}=0$ and $v_{y}=2 \pi R_{E} / T$ where $T=31556925.99 \mathrm{~s}$ as the time for a single orbit. The velocity Verlet algorithm was used throughout. A perfect simulation would return the Earth precisely to its original position after one year, that is to $\left(R_{E}, 0\right)$.

The distance error, $\Delta r$, of the calculated distance of the Earth from the starting position after one year forms a measure of simulation accuracy. Figure 1 presents $\Delta r$ as a function of time step $\Delta t$ using single-precision and double-precision arithmetic. For a given $\Delta t$, the quantity $\Delta r$ was found by locating time step $j$ such that $y_{j}<0$ and $y_{j+1}>0$, completing a linear interpolation to find the value of $x=x_{0}$ when $y=0$ and setting $\Delta r=\left|x_{0}-R_{E}\right|$. The absolute value is used so that the data can be presented logarithmically.

First, examine the results using double-precision arithmetic starting at top right of Figure 1 where $\Delta t \approx 22 \mathrm{hrs}$. As the simulation time step is reduced, $\Delta r$ exhibits an approximately linear reduction (with a gradient of +2 ) to a minimum indicated by the symbol within a circle. One would expect the simulation accuracy to continue to improve as the simulation time step is reduced further, but this is not the case. In fact, as $\Delta t$ is further reduced, simulation accuracy decreases (with a gradient of -2). The change in gradient is indicative 


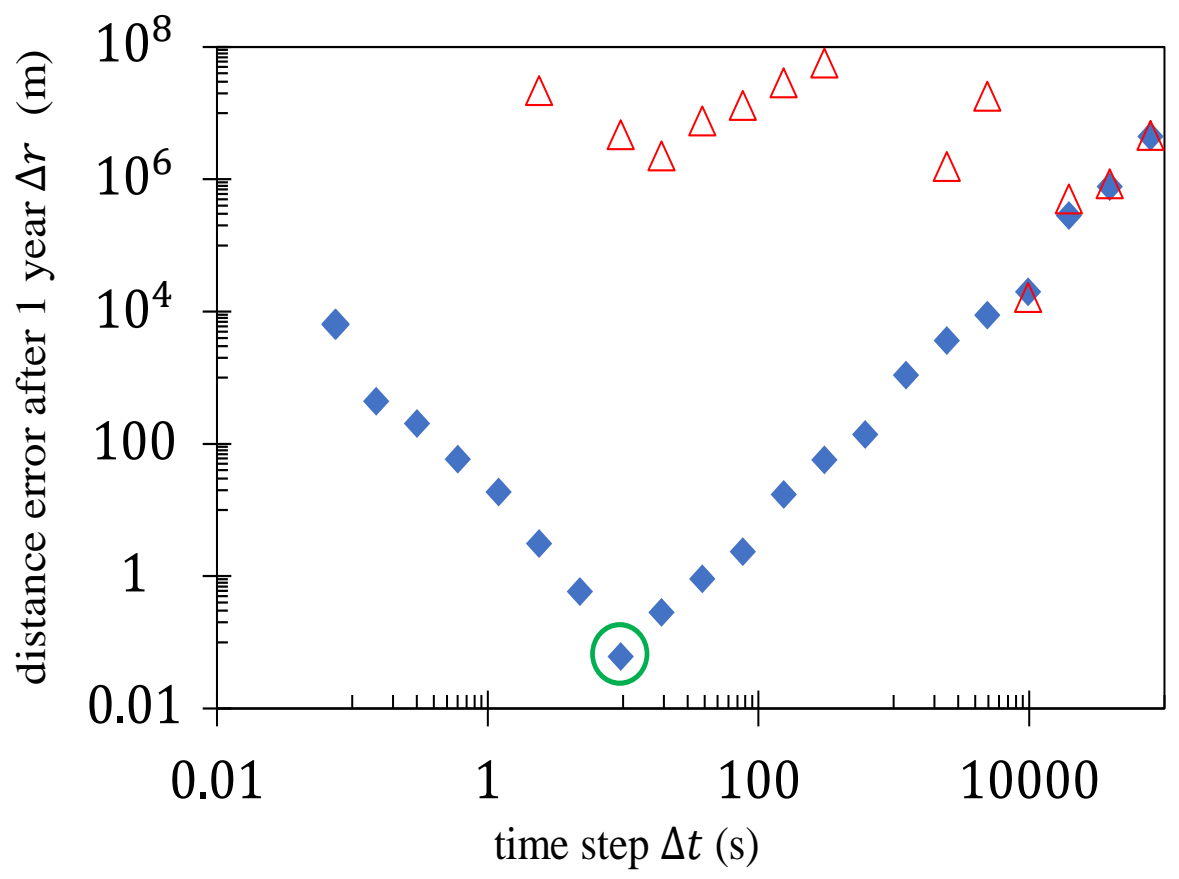

FIG. 1. The deviation of the numerical simulation from a precise circular Earth orbit after one year is plotted as a function of the simulation time step $\Delta t$. The numerical simulation was executed using double precision $(\diamond)$ and single precision $(\triangle)$. The circle highlights the optimum performance of the simulation.

of a change in the source of error. The worsening of the accuracy beyond (in this simulation) $\Delta t \approx 20 \mathrm{~s}$ is due to the influence of the 15 significant figure precision limit of double-precision arithmetic. The coordinate $x$ at successive time steps for the optimum time step $\Delta t \approx 20 \mathrm{~s}$ is found to differ at the $12^{\text {th }}$ significant figure. Floating-point error at the $15^{\text {th }}$ significant figure accumulated through repeated calculations adversely affects the accuracy of the orbit trajectory for $\Delta t<20 \mathrm{~s}$ and worsens as the double-precision limit is reached at the shortest time steps.

The results using single-precision floating-point numbers also show improving accuracy of the orbital distance as $\Delta t$ decreases from 22 hours, but the improvement is lost below about $\Delta t \approx 10000 \mathrm{~s}$. Indeed a student may be baffled to find that reducing the time step to $\Delta t=5000 \mathrm{~s}$ or further to $2000 \mathrm{~s}$ yields deteriorating agreement with the sought-after exact result. Figure 1 demonstrates that normally unimportant errors such as floating point adversely impact the accuracy of the model for time steps shorter than an optimum value.

The Verlet algorithm is accurate to second order in velocity and to the third order in posi- 


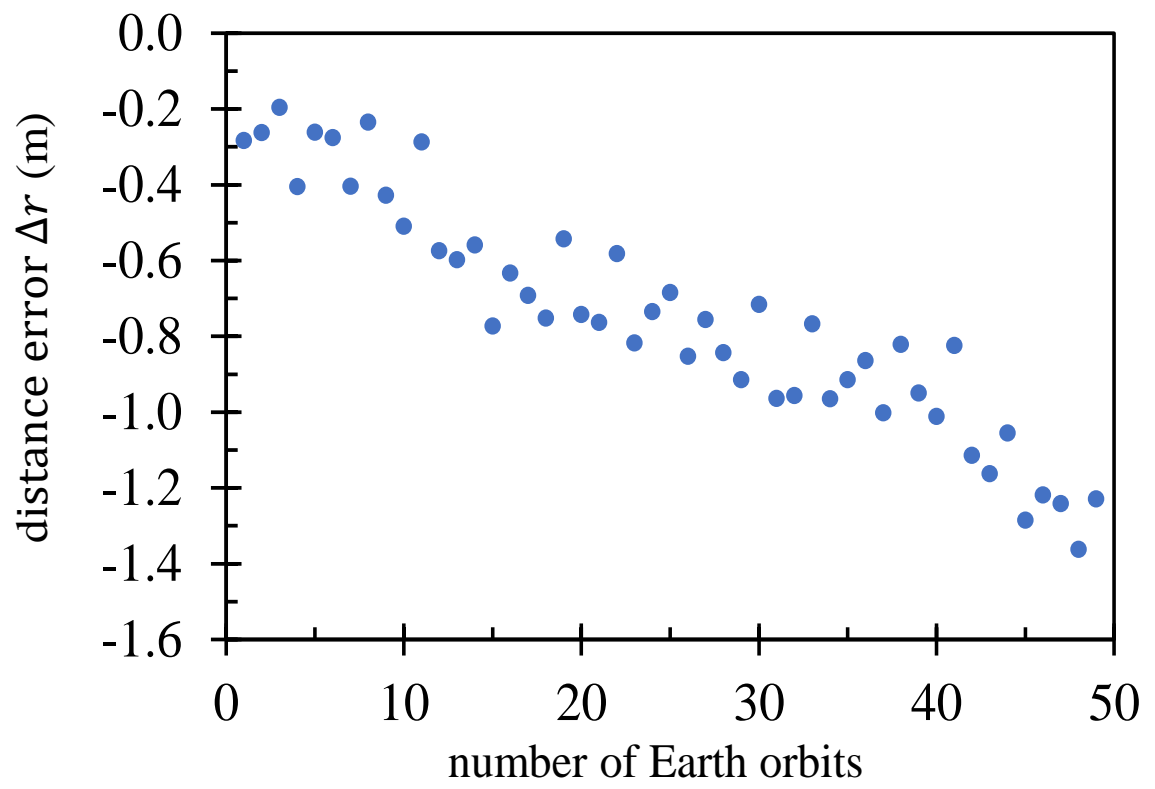

FIG. 2. The orbital deviation distance $\Delta r$ of the numerical simulation from an idealised circular orbit of a model Earth is presented as a function of the number of orbits. The optimum time step of $19.3 \mathrm{~s}$ and a velocity Verlet algorithm are used.

tion, but its impressive performance arises because it is an energy-conserving, or symplectic, integrator. The quality of the Verlet algorithm is illustrated in Fig. 2 which takes the optimum time step $\Delta t=19.3 \mathrm{~s}$ and presents the orbital radius deviation $\Delta r$ as a function of the number of orbits of the Earth around the Sun. The fractional change in the total energy of the Earth is about $10^{-12}$ in each orbit whereas changes of typically $10^{-4}$ per orbit are seen with the non-Verlet approach. ${ }^{19}$ The energy-conserving property of Verlet provides orbits accurate to a remarkable $20-40 \mathrm{~cm}$ for the first 10 orbits with a linear deterioration of accuracy to about $1.4 \mathrm{~m}$ after 50 orbits.

Once the Earth model has been validated, students undertake a second simulation for the orbit of a fictional asteroid. The parameters are the same as the Earth model except for the initial conditions of position and velocity which are supplied by the instructor with associated uncertainties. ${ }^{17}$ The objective now is to calculate the minimum asteroid-Earth distance to assess the risk the asteroid poses to the Earth. Students therefore quantify sources of uncertainty to identify the primary source of error. The results summarised in Figs. 1 and 2 demonstrate that algorithmic error is negligible if the Verlet algorithm is used, and that floating-point error can be avoided with careful choice of time step and significant 
figure precision. Other model shortcomings, such as the neglect of the gravitational pull of Jupiter or the rotation of the Earth about the Earth-moon center of mass can be estimated. Students may then repeat simulations varying the initial conditions of position and velocity of the asteroid to reflect their uncertainties to establish that this is the primary source of error for the calculation of the minimum asteroid-Earth distance.

\section{B. Integration by computation}

The second example of loss of accuracy due to the limited accuracy of floating-point numbers emerges from numerical integration. Consider the integral

$$
G(t)=\int_{0}^{\infty} e^{-D t z^{2}} J_{3 / 2}(z) z^{-1} d z
$$

where $J_{3 / 2}(z)$ is a Bessel function of the first kind, $D$ is a positive constant and $z$ is the dimensionless integration variable. The function $G(t)$ that we are asked to determine is called a dipolar correlation function which is part of a calculation of the nuclear magnetic resonance response of a proton-bearing fluid, such as water. The Gaussian function is the diffusion term with $D$ related to the diffusion coefficient of the water and may be written $D=\tau^{-1}$ where $\tau$ is a time constant. The remaining terms describe the interaction of pairs of proton spin dipoles. The physics need not concern us here. We are interested in finding a method of evaluating the integral which is required as a function of time for the range $t^{*}=t / \tau=0 \ldots 100$ where $\tau=1 \mathrm{ps}$ and $t^{*}$ is a dimensionless time.

A student may opt for numerical integration, providing an opportunity to compare numerical integration schemes such as the trapezium method, Simpson's rule and Gaussian quadrature. The Bessel function is supplied as a scipy.special function in Python and does not pose a difficulty for calculation. Indeed, this specific integral provides an opportunity to expose students to special functions in Python or equivalents in other programming languages. Monte Carlo integration techniques, which are used widely to determine integrals in nuclear physics (albeit for far more complex integrations) could be explored, but to use this technique a reasonable maximum value for the integrand would be need to be found at each $t^{*}$. A third approach would be to use an algebraic computing package such as Mathematica or Maple. Results are accurate but restrictively lengthy for some values of $t^{*}$. Alternatively, students may attempt an analytical solution. The integral is found in a 


$$
G\left(t^{*}\right)=\frac{3 \sqrt{\pi}}{4 \sqrt{2}} t^{*} \sum_{n=0}^{\infty} a_{n} \quad a_{0}=1, \quad a_{n}=a_{n-1} \frac{-2 t^{*}}{3+2 n} .
$$
written

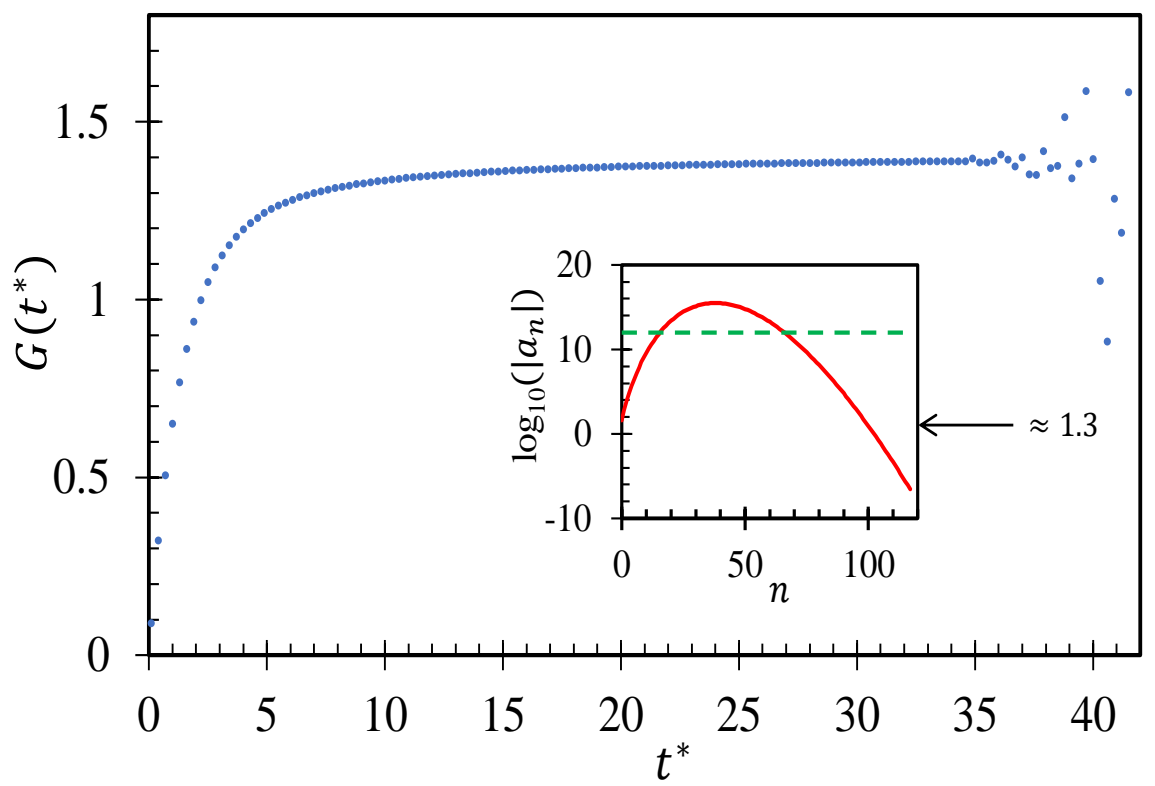

FIG. 3. The results for a function $G\left(t^{*}\right)$ as calculated using Eq. (6) are presented (•) as a function of the dimensionless time $t^{*}$. The summation converges but yields incorrect results if $t^{*} \gtrsim 36$. The inset figure presents $\log \left(\left|a_{n}\right|\right)$ against $n$ for $t^{*}=40$ (—). The line (- - - -) is set at $1.5 \times 10^{12}$. The values of $\left|a_{n}\right|$ above this line indicate that individual terms of the summation are more than $10^{12}$ larger than the value of $G\left(t^{*}=40\right) \approx 1.3$.

standard book of tables of integrals. ${ }^{21,22}$ After some trivial simplifications, $G\left(t^{*}\right)$ may be 
3 many values of $a_{n}$ are more than 12 orders of magnitude greater than the correct final value of $G\left(t^{*}\right)$. Thus, in Eq. (6) pairs of terms of similar magnitude are added, and since one of each pair is positive while the other is negative, the addition leads to errors at significant figures in the region of the final result.

This example is an illustration of subtractive cancellation which is discussed in some detail by Landau et al. who show that, for certain simple problems (such as solving a quadratic equation), the subtractive cancellation problems can be avoided. ${ }^{4}$ In the present example, it is instructive to execute a standard numerical integration approach to the determination of the integral in Eq. (5) to run alongside the summation approach in Eq. (6). A student could assess the shortcomings of each approach. In fact, for this specific example, accurate results can be obtained across the full range of $t^{*}$, with each method being appropriate for a different range of $t^{*}$.

\section{PROPAGATION OF FLOATING-POINT REPRESENTATION ERROR}

\section{A. The Bernoulli orbit}

The Bernoulli orbit is an example of a chaotic dynamical system that can be expressed by a simple iterative mathematical algorithm. A chaotic dynamical system is acutely sensitive to its initial conditions. Examples of many-particle chaotic systems include molecular dynamics, climate modeling and, of course, the motion of an asteroid through the solar system. Most dynamical computer simulations in science are fundamentally chaotic. Often, chaotic behavior is judged to be an attribute of a dynamical simulation as one desires to compute the statistical average of physical quantities. For example, the calculation of the diffusion coefficient of molecules in a liquid depends on how far each molecule has moved from its initial position in a given time period. The square of the distance moved is computed for each molecule in a large box, and the statistical average of these squares is then used to calculate the diffusion coefficient.

Boghosian, Coveney and Wang demonstrated that, under certain circumstances, statistical results from numerical simulations could include a significant component due to floating-point representation error. ${ }^{23}$ The conclusions drawn from chaotic numerical simulations (climate modeling, molecular dynamics) could therefore include a contribution not 
associated with the phenomena under investigation. The authors used Bernoulli orbits to illustrate the effect that the propagation of floating-point representation error can have.

We illustrate this effect with the simplest and most impactful example. A Bernoulli orbit is generated as a sequence of numbers such that

$$
x_{i+1}=\sigma x_{i} \quad \bmod 1
$$

where $\sigma$ is a Bernoulli number. For illustration, $\sigma=2$ is chosen. A number between 0 and 1 is selected as the first value of the sequence, say $x_{0}=0.6$. Subsequent values of $x_{i}$ in the sequence are produced by repetition of Eq. (7), so that $x_{1}=0.2, x_{2}=0.4, x_{3}=0.8$, $x_{4}=0.6$ with the cycle then repeating. This is referred to as a Bernoulli orbit and this orbit is illustrated in Figure 4.

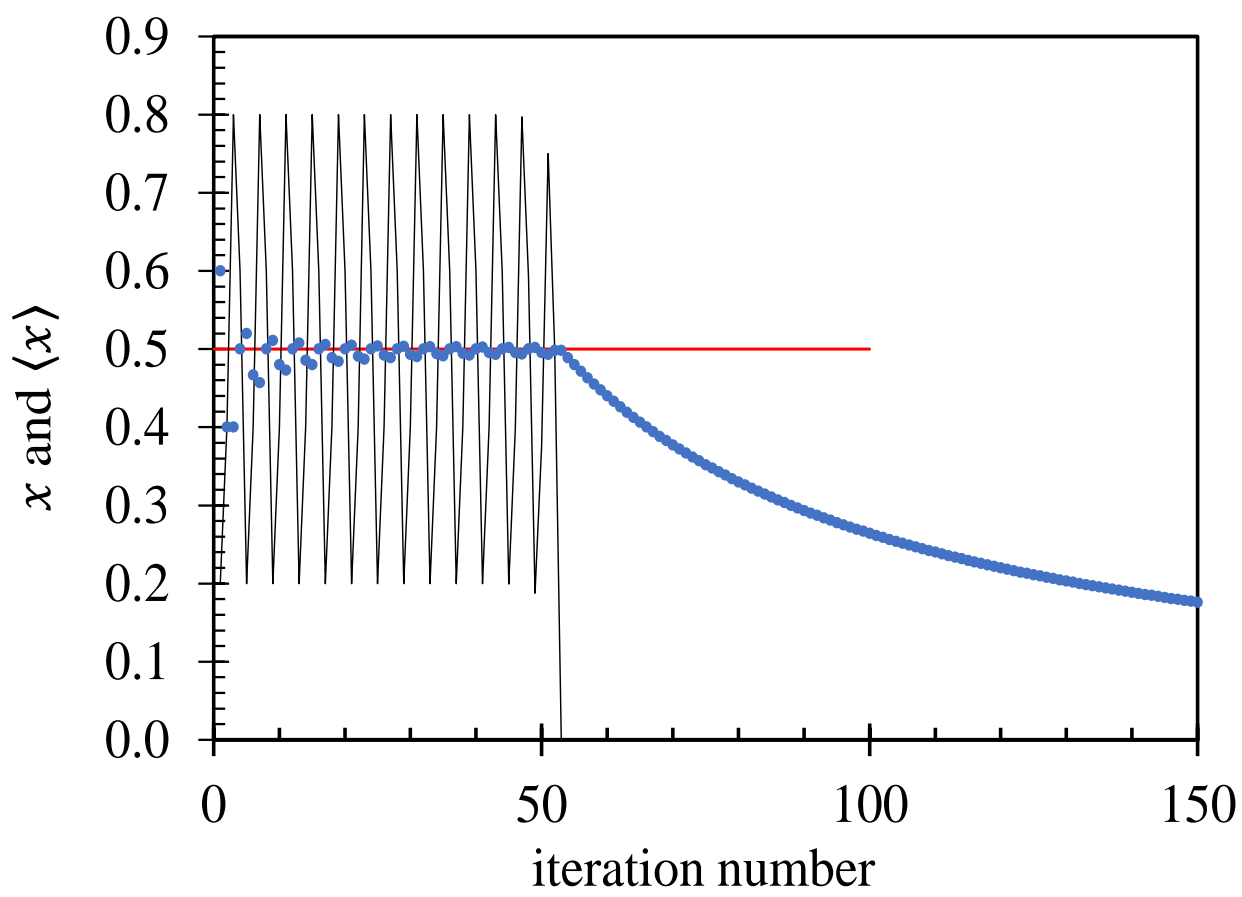

FIG. 4. A Bernoulli orbit is presented using Eq. (7) with $\sigma=2$ and $x_{0}=0.6(-)$. The orbit falls to zero at $i=53$. $\langle x\rangle$ is presented as a running average $(\bullet)$. The horizontal red line represents the exact value of $\langle x\rangle$.

The mean value $\langle x\rangle$ is a statistical property that can be measured from the sequence of numbers of the Bernoulli orbit. The exact value $\langle x\rangle=0.5$ is easily calculated and is represented as the horizontal solid line in Figure 4. An eight line Matlab code was written 
to evaluate $\langle x\rangle$ as a running average and the results are displayed in Figure $4 .{ }^{19}$ Unexpectedly, the computation yields $\langle x\rangle \rightarrow 0$ as $i \rightarrow \infty$ rather than the correct average of 0.5 .

The explanation for the incorrect determination of $\langle x\rangle$ is revealed in Figure 4. The Bernoulli orbit breaks down at $i \approx 50$ such that $x_{i}=0$ for $i>53$. Boghosian, Coveney and Wang explain that floating-point representation error starting at about the $15^{\text {th }}$ decimal place propagates until it impacts significantly the value of $x_{i}$ at the first decimal place. ${ }^{23}$ This feature is most extreme for $\sigma=2$ because of the binary digit representation of numbers. The error propagates one decimal place per orbit, resulting in the value of $x_{i}$ falling to zero in the $14^{\text {th }}$ orbit.

In this example, it is clear that the numerical computation using a Bernoulli number $\sigma=2$ gives incorrect values. Boghosian, Coveney and Wang undertook calculations with different values of $\sigma$ and established that floating-point error propagation may significantly contribute to the measured statistical properties of the simulation data, $\langle x\rangle$ for instance, but the contribution cannot easily be quantified..$^{23}$ Their conclusion was that it is possible that floating-point propagation may contribute to the statistical property being measured in dynamical simulations.

\section{B. Cellular automata - the game of life}

Cellular automata are widely used in undergraduate physics courses to educate students in elementary programming and for project work. Cellular automata are straightforward to code based on simple rules and therefore allow individual students to undertake personal project work in areas of physics such as diffusion, magnetism, thermodynamics, econophysics, social physics and finance, to name but a few.

Conway's classic game of life is a cellular automata in which an individual cell is either alive (1) or dead (0) but can change at each generation according to rules which are based on the sum of the liveness values of its eight surrounding cells. ${ }^{24}$ A "semi-quantum" version of the game of life was introduced by Flitney and Abbott in 2008 in which cells are represented as qubits with modified evolutionary rules. ${ }^{25}$ Game-of-life projects have proved substantially the most popular projects for undergraduate students in our Physics Department. These multifaceted projects provide the opportunity for students to capitalise on their coding expertise, to learn about qubits and their evolution based on interactions with the local 
environment, and to link aspects of quantum computation with concepts in the life sciences including quantum biology.

The second example of the impact of the propagation of floating-point representation error emerges from a cellular automata variant called the "semi-classical game of life", or SCGOL. ${ }^{26}$ The SCGOL game is based on the scheme established by Flitney and Abbott but with real qubits. The SCGOL is typical of a variety of cellular automata used for project work due to the simplicity of the rules (hence coding) leading to complex behaviors that provide opportunities for individual research. ${ }^{27,28}$ Various opportunities for project work in physics based on the SCGOL have already been suggested..$^{26}$ The SCGOL is appealing, not because the propagation of floating-point representation error is responsible for chaos-like behavior or that the source of the error is unexpected, but because the effect is sudden and dramatic and therefore provides in opportunity to test directly the assertion of Boghosian, Coveney and Wang that propagation of floating-point representation error may impact the statistics of simulation output data. ${ }^{23}$

The space of the SCGOL comprises a $49 \times 49$ grid of cells, each of which is assigned a number between zero and 1 to represent its "liveness". A value of 0 indicates that the cell is dead, a 1 indicates a fully live cell, and a value in between indicates a part-live cell. The initial state of the system comprises a $3 \times 3$ object placed at the centre of an otherwise dead space as shown in Figure 5. The corner cells of this object are set to 0.58 , the central cell is dead (0) and the remaining cells are fully live (1). Each cell evolves at each time step on the basis a set of evolutionary rules that depend on the sum of the liveness of the eight surrounding cells, $A$, called the Moore neighborhood of the cell. Full details of the game and the evolutionary rules may be found in Faux et al. ${ }^{26} \mathrm{~A}$ sequence of four images at time steps 0, 20, 60, and 100 is presented in Figure 5. The system initially evolves with the expected four-fold symmetry but, after about 60 generations, the system is seen to lose its symmetry and the evolution appears "chaotic" beyond 80 generations. A video of the full evolution shown in Figure 5 is presented as supplementary material. ${ }^{29}$

The loss of symmetry is due to the propagation of floating-point representation error but the source of error was difficult to identify. Each cell evolves according to a rule based on the sum of the liveness of its eight neighboring cells. The four-fold symmetry of the initial 

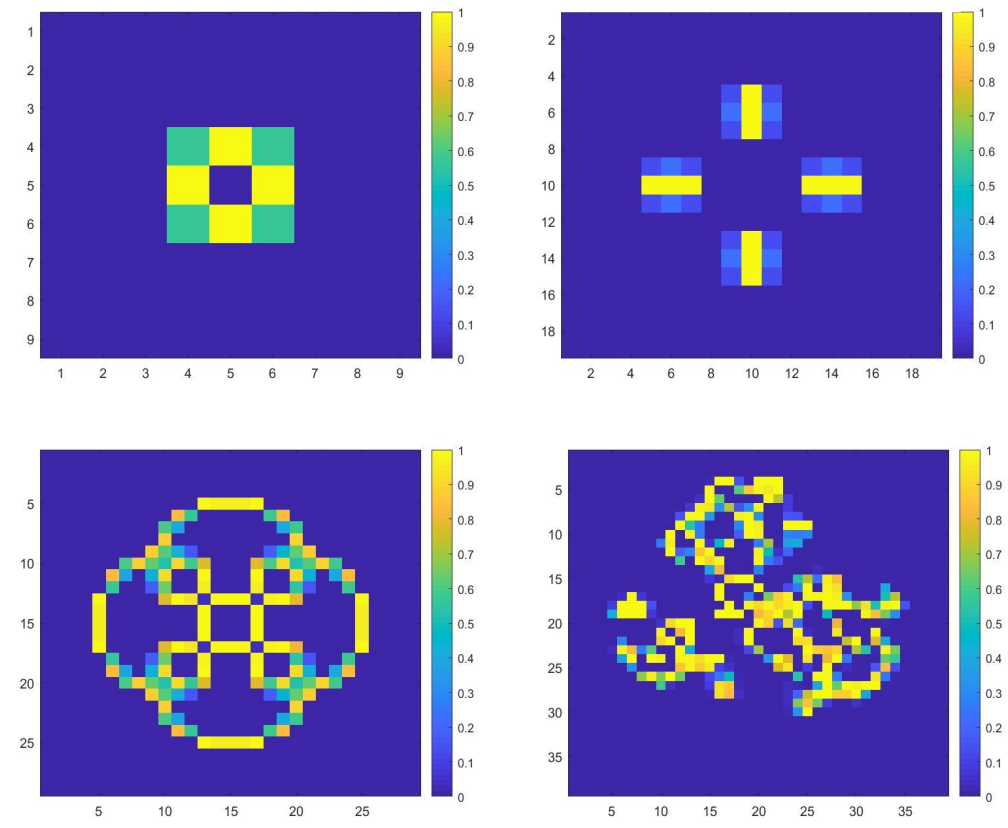

FIG. 5. The semi-classical game of life starts with a $3 \times 3$ "seed" object with perfect four-fold symmetry (top left). The object changes at each generation according to a set of evolutionary rules that should preserve the symmetry. The images show the evolution after 20 (top right), 60 (bottom left) and 100 (bottom right) generations.

"seed" object at top left of Figure 5 should ensure that the evolution develops with perfect four-fold symmetry. A cell in one quadrant should have an equivalent cell in each of the three remaining quadrants which possesses precisely the same liveness value. Each of the four equivalent cells should have an identical set of (up to) eight values of liveness in its Moore neighborhood, and therefore an identical value of the parameter $A$, which is the sum of these livenesses.

The first breach of symmetry is detected in the third generation of the system. Table III lists the five non-zero neighboring cell liveness values for two equivalent cells in different quadrants. The five numbers in one list are identical to the five numbers in the second list to 18 significant figures confirming that each pair of identical numbers have precisely the same bit strings. Computing the sum of the five numbers in each list involves adding five numbers with identical bit strings. However, although the results of the two summations appear identical when rounded to 15 significant digits, the summations are different from the $16^{\text {th }}$ decimal place. The difference arises due to the order in which the summations are executed. Due to the arrangement of the surrounding cells in the two cases, the second and third numbers of the summation for one cell become the third and second numbers, respectively, 
TABLE III. The table presents a summation of five numbers that appears in a cellular automaton called the semi-classical game of life. ${ }^{26}$ Two "identical" sums are executed with the order of addition indicated.

\begin{tabular}{rcc}
\hline \hline Order & Sum 1 & Sum 2 \\
\hline 1 & 0.624020061714517960 & 0.624020061714517960 \\
2 & 0.624020061714517960 & 0.136759019438273594 \\
3 & 0.136759019438273594 & 0.624020061714517960 \\
4 & 0.994497833431808753 & 0.994497833431808753 \\
5 & 0.994497833431808753 & 0.994497833431808753 \\
$A$ & 3.373794809730927380 & 3.373794809730926936 \\
\hline \hline
\end{tabular}

of the summation for the other cell. In simple terms, $(a+b)+c \neq(a+c)+b$. This leads to a difference in $A$ that gradually propagates leading to the observed symmetry-breaking.

The SCGOL cellular automaton may now be used to test the proposal of Boghosian, Coveney and Wang that statistical averages from a simulation may include a contribution due to floating-point error propagation. ${ }^{23}$ A SCGOL simulation of a $500 \times 500$ system starting with an initial randomly-generated configuration of liveness was evolved for 30 generations with the final liveness configuration written to file to just six decimal places. The simulation is too short to allow the propagation of floating-point errors to appear in the first six decimals. The end point of this simulation is then used as the initial configuration for a fresh simulation run for 130 generations. The mean and standard deviation of the liveness was determined for generations 10 to 40 inclusive and for generations 100 to 130 inclusive. The first sample is too short for floating-point errors to have impacted the statistics but the second is long enough for floating-point errors to have propagated through to all cells in the system. The results for the mean and standard deviation are the same within the expected statistical uncertainty suggesting floating-point propagation error does not impact these particular results.

\section{UNEXPECTED CONSEQUENCES OF ROUNDING ALGORITHMS}

Rounding algorithms truncate a decimal number to a specified number of decimal places. Rounding to zero decimal places converts a decimal to an integer. Table I, however, shows that the digit after the decimal point is different, such as 0.3 for example which presents as 0.299999999... Simple rounding to one decimal place will yield 0.2 rather than 0.3. Failure 
TABLE IV. The table presents example outputs from the round command from Python and Matlab.

\begin{tabular}{ccc}
\hline \hline command & Python & Matlab \\
\hline round $(2.605,2)$ & 2.60 & 2.61 \\
round $(2.615,2)$ & 2.62 & 2.62 \\
round $(2.625,2)$ & 2.62 & 2.63 \\
round $(2.635,2)$ & 2.63 & 2.64 \\
round $(2.645,2)$ & 2.65 & 2.65 \\
round $(2.655,2)$ & 2.65 & 2.66 \\
round $(2.665,2)$ & 2.67 & 2.67 \\
round $(2.675,2)$ & 2.67 & 2.68 \\
round $(2.685,2)$ & 2.69 & 2.69 \\
round $(2.695,2)$ & 2.69 & 2.70 \\
\hline \hline
\end{tabular}

to take measures to avoid rounding errors can lead to unexpected results, and even tragic death as in the case of the patriot missile disaster. ${ }^{30}$

\section{A. The round command}

The spreadsheet package EXCEL uses the commands ROUNDUP, ROUNDDOWN and ROUND for rounding real numbers to the higher, lower and nearest integer respectively. Languages Python and Matlab both employ equivalent functions and use the round command with flexibility, so that rounding can be specified to a number of decimal places. For example, the command round $(2.678,2)$ produces the result 2.68 in both languages. An excellent review of the use of truncation and rounding in Python is provided by Amos and in the documentation of the Python collaboration. ${ }^{31,32}$ Table IV shows the output from using the round command on a sequence of decimals in each of Python and Matlab. ${ }^{19}$ Matlab yields the outcomes one might expect, that is, the final digit " 5 " is rounded upwards in all cases, whereas Python yields unpredictable outcomes.

The explanation for the results produced by Python is provided by Table I. The binary representation of 2.635 appears as 2.634999999999999787 to 18 decimal places so the command round $(2.635,2)$ rounds down to 2.63 . However, the floating-point number 2.635 is output as 2.634999999999999787 in both Python and Matlab as one would expect (since both program languages use the same number of bits to represent the mantissa). Indeed, round $(2.635,2)$ and round $(2.634999999999999787,2)$ both round to 2.64 in Matlab! The 

correction.

\section{B. Timing devices}

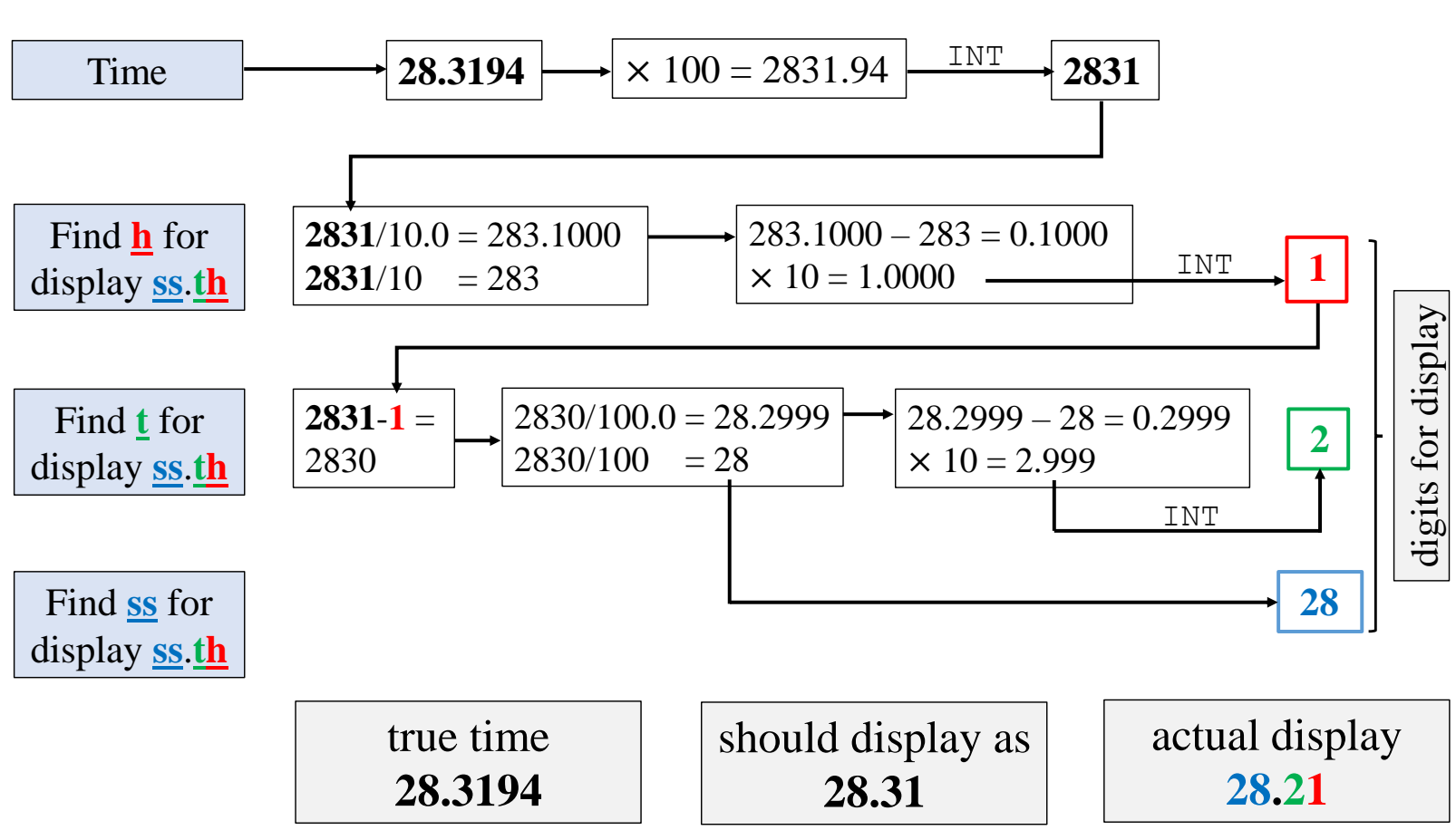

smallest number that rounds up to 2.64 in Matlab is 2.634999999999999565, which is exactly $1 / 2^{52}$ less than 2.634999999999999787. It appears that two final bits $\left(1 / 2^{51}\right)$ are added to a number in Matlab to provide the "expected" rounding and constitutes a form of error

By far the most widespread use of real-to-integer conversion is for digital displays as, for example, in digital clocks and timing devices. Most digital timing devices rely on the oscillations of a quartz crystal for timing and the National Institute of Standards and Technology (NIST) guide provides an excellent background to their function. ${ }^{33}$ The quartz crystal is normally set to oscillate at $32768=2^{15} \mathrm{~Hz}$ so that each "tick" is of duration $3.0518 \times 10^{-5} \mathrm{~s}$. A circuit comprising flip-flop digital dividers counts the number of oscillations between a start signal and a stop signal and stores the count in a binary register. ${ }^{34,35}$ The number of counts is multiplied by $3.0518 \times 10^{-5}$ to obtain a high-precision time in seconds accurate to approximately $0.0001 \mathrm{~s}$ (three ticks of the quartz oscillator). There are two steps in the

FIG. 6. Starting with an accurate time of $28.3194 \mathrm{~s}$, the flow diagram illustrates how this time is converted into the integer numbers for display to two decimal places using a standard procedure. ${ }^{36}$ The INT command is equivalent to a truncation and takes the lower integer. 
process of producing a lower-precision time for digital display. In the first step, the quartz oscillations are counted and then multiplied by $3.0518 \times 10^{-5}$ to generate a floating-point time in seconds accurate to four decimal places. The second step requires display software to isolate the integer digits for display. Stopwatches normally display times in the $\mathrm{m}$ : ss. th format (minutes, seconds, tenths and hundredths) or the shortened ss. th format if $\mathrm{m}$ is zero. Suppose, for illustration, a stopwatch produces a time of $28.3194 \mathrm{~s}$ to be displayed in the ss.th format. One process of converting a binary field to a digital display is described in an IBM Knowledge Center document. ${ }^{36}$ This procedure isolates the relevant integer digits to present the three integers ss, $\mathrm{t}$ and $\mathrm{h}$. The procedure is designed to display $28.3194 \mathrm{~s}$ as 28.31 , that is the number is truncated at two decimal places and not rounded up.

The procedural steps for converting a stopwatch time to a digital display are illustrated in Figure $6 .{ }^{36}$ The algorithm was coded using FORTRAN and all the numerical values are faithfully reproduced in the flow diagram. ${ }^{19}$ The time $28.3194 \mathrm{~s}$ displays as 28.21 instead of 28.31 because a rounding error produces the digit 2 in the display for the tenths position instead of 3. A difference of $0.1 \mathrm{~s}$ is of similar order to the expected variation of human reaction time for a stopwatch. ${ }^{37}$ The presentation of the incorrect display digit is not a property of the particular algorithm used here. Any truncation algorithm would have the same issues of rounding.

In 2019, we estimated the characteristic human reaction time for a manually-operated timing device from 4304 swimming competition race times obtained where races were started electronically and finished both electronically and manually. ${ }^{37}$ We found that the reaction time for a single manual time measurement was modeled by a normal distribution with a mean of $0.11 \mathrm{~s}$ and a standard deviation of $0.07 \mathrm{~s}$. These results represent the uncertainty associated with human reaction time and can be used for error analysis in physics experimentation where manual timing is used. If rounding errors are uncorrected in stopwatches, stopwatch timing provides an additional source of uncertainty.

This article provides undergraduates with examples of the effects of floating-point errors that are interesting and appropriate at undergraduate level. Strategies to reduce or avoid floating-point error are illustrated. The examples can be used to instigate discussion and to form the focus of student projects. It should be noted that these errors may sometimes have real world consequences. In an article in the Notes and Discussion section, we provide 
compelling experimental evidence that rounding errors are indeed present in times recorded by stopwatches. It is shown that rounding error can be sufficiently large to reverse the ranking of two athletes in a close race. ${ }^{38}$

* d.faux@surrey.ac.uk

1 Institute of Electrical and Electronics Engineers. Computer Society. Standards Committee and David Stevenson, IEEE standard for binary floating-point arithmetic (IEEE, 1985).

2 J. H. Wilkinson, in Rounding Errors in Algebraic Processes, edited by A. A. Grau, (Society for Industrial and Applied Mathematics, 1966).

3 James H Wilkinson, "Error analysis of floating-point computation," Numerische Mathematik 2 (1), 319-340 (1960)

4 Rubin H. Landau, Jose Paez, Manuel José Páez Mejía, and Cristian C. Bordeianu. A survey of computational physics: introductory computational science, Princeton University Press, 2008.

5 Linda Grandell, Mia Peltomäki, Ralph-Johan Back and Tapio Salakoski, "Why complicate things? Introducing programming in high school using Python", Proceedings of the 8th Australasian Conference on Computing Education 52, 71-80 (2006).

6 Athanassios Jimoyiannis and Vassilis Komis, "Computer simulations in physics teaching and learning: a case study on students' understanding of trajectory motion," Comput. Educ., 36 (2), 183-204 (2001).

7 Peter Junglas, "Simulation programs for teaching thermodynamics," Global J. of Engng. Educ., 10 (2), 175-180 (2006).

8 Rubin Landau, "Simulation programs for teaching thermodynamics," Comput. Phys. Commun., 177 (1-2), 191-194 (2007).

9 Elvira Martinez, Mercedes Florez and Jose Amaya, "Simulations as a new physics teaching tool," Comput. Appl. Eng. Educ., 18 (4), 757-761 (2010).

10 Jesse M Kinder and Philip Nelson, A Student's Guide to Python for Physical Modeling: Updated Edition, (Princeton University Press, Princeton, 2018).

11 Philip C Nelson and Tom Dodson, A Student's Guide to Matlab for Physical Modeling, see https://works. bepress.com/philip-c-nelson/104.

12 Claudio Rebbi, "A project-oriented course in computational physics: Algorithms, parallel com- 
puting, and graphics," Am. J. Phys. 76 (4), 314-320 (2008).

13 Todd Timberlake and Javier E. Hasbun, "Computation in classical mechanics," Am. J. Phys. 76 (4), 334-339 (2008).

14 David M. Cook, "Computation in undergraduate physics: The Lawrence approach," Am. J. Phys. 76 (4), 321-326 (2008).

15 Andy Buffler, Seshini Pillay, Fred Lubben and Roger Fearick, "A model-based view of physics for computational activities in the introductory physics course," Am. J. Phys. 76 (4), 431-437 (2008).

16 Ruth Chabay and Bruce Sherwood, "Computational physics in the introductory calculus-based course," Am. J. Phys. 76 (4), 307-313 (2008).

17 The instructions provided to students is provided as a Word document as supplementary material at [URL will be inserted by AIP]

18 "Decimal fixed point and floating point arithmetic" in The Python Standard Library, available at https://docs.python.org/3/library/decimal.html [Accessed: February 2021].

19 All computer codes referred to are provided as supplementary material at [URL will be inserted by AIP].

20 A summary of the many forms of Verlet integration algorithms may be found at the wikipedia site Verlet Integration, available at https://en.wikipedia.org/wiki/Verlet_integration [Accessed: February 2021].

21 Milton Abramowitz, Irene A Stegun, Handbook of mathematical functions with formulas, graphs, and mathematical tables, Dover Publications, Inc., New York (Apr 2014).

22 I S Gradshteyn and I M Ryzhik, Table of integrals, series, and products, Daniel Zwillinger and Victor Moll (eds.), Elsevier, New York, Eighth edition, (2015).

23 Bruce M. Boghosian, Peter V. Coveney and Hongyan Wang, "A new pathology in the simulation of chaotic dynamical systems on digital computers," Adv. Theory Simul. 2 (12), 1900125, 1-8, (2019).

24 Martin Gardner, "Mathematical Games - The fantastic combinations of John Conway's new solitaire game "life" ", Sci. Am. 223, 120-123 (1970) DOI=10.1038/scientificamerican0770-117

25 Adrian P. Flitney and Derek Abbott, "A Semi-Quantum Version of the Game of Life" in Quantum Aspects of Life, pp 233-250, published by World Scientific (2008).

26 David A. Faux, Mayank Shah and Christopher Knapp, "Games of life", Am. J. Phys. 88, 
371-378 (2020).

27 Apinya Dhatsuwan and Monamorn Precharattana, "BLOCKYLAND: A cellular automatabased game to enhance logical thinking," Simulat. Gaming 47 (4), 445-464 (2016).

28 G. Faraco, Pietro Pantano and Rocco Servidio, "The use of cellular automata in the learning of emergence," Comput. Educ. 47 (3), 280-297 (2006).

29 A video of the evolution of the "seed" object using the rules of the semi-classical game of is supplied a supplementary material at [URL will be inserted by AIP]

30 Douglas N. Arnold The patriot missile failure, http://www-users.math.umn.edu/ arnold/ disasters/patriot.html. [Accessed: February 2021].

31 David Amos, How to round numbers in Python, realpython.com/python-rounding [Accessed: February 2021].

32 Python Documentation: The Python Tutorial 15, Floating Point Arithmetic: Issues and Limitations, https://docs.python.org/3/tutorial/floatingpoint.html [Accessed: February 2021].

33 Jeff C. Gust, Robert M. Graham and Michael A. Lombardi, Stopwatches and Timer Calibrations, NIST Recommended Practice Guide, (2009 edition).

34 Michael Robbins, Electronic Clocks and Watches, Howard W. Sams Co., Indianapolis, IN, (1975).

35 J. R. Vig, Stopwatches and Timer Calibrations, NIST Recommended Practice Guide, (1992).

36 Converting binary fields to time of day format, IBM Knowledge Center, at https://www.ibm.com/support/knowledgecenter/en/SSLTBW_2.1.0/com.ibm.zos.v2r1. ieag200/cnvttime.htm [accessed February 2021]

37 David Faux and Janet Godolphin, "Manual timing in physics experiments: Error and uncertainty", Am. J. Phys. 87 (2), 110-115 (2019).

38 David Faux and Janet Godolphin, "The floating point: rounding error in timing devices", Am. J. Phys. [editor to insert] (2021). 